\title{
HEADACHES AND SYPHILIS DO NOT ALWAYS MEAN NEUROSYPHILIS: A TRICKY CASE OF SECONDARY SYPHILIS.
}

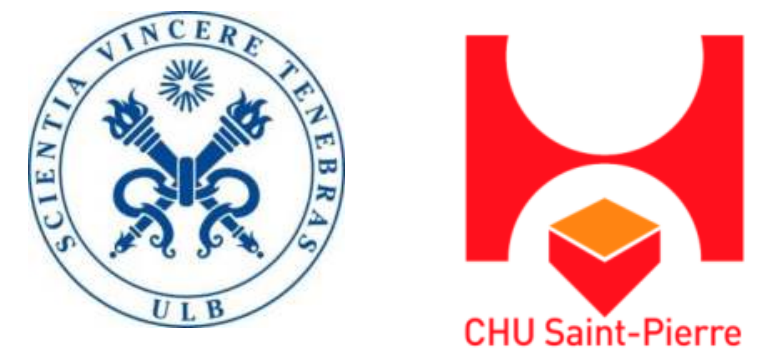

J. Krygier ${ }^{a}$, U. Sass ${ }^{a}$, A. Kolivras $^{a}$, I. Ferreira ${ }^{a}$, M. Tondeur ${ }^{b}$, A. Libois ${ }^{c}$.

a Departments of Dermatology and Dermatopathology, Saint-Pierre, Brugmann and Queen Fabiola Children's University Hospitals, Université Libre de Bruxelles. Brussels, Belgium.

${ }^{b}$ Department of Radioisotopes, CHU Saint-Pierre, Université Libre de Bruxelles, Brussels, Belgium.

c Department of Infectious Diseases, CHU Saint-Pierre, Université Libre de Bruxelles, Brussels, Belgium.

\section{CASE REPORT:}

A 46-year-old HIV-positive man, treated with an undetectable viral load, consulted for an eruption of multiple large erythematosquamous plaques accompanied by headaches and fever since 1 month. Skin biopsy was characterized by the presence of a plasma cell-rich granulomatous infiltrate in which treponema immunohistochemistry was positive. Blood serology revealed a positive treponemic test and a Rapid Plasma Reagin test increased at 1/256. His last negative syphilitic test dated from 15 months earlier. Diagnosis of secondary syphilis was made. Neurosyphilis was ruled out with a negative lumbar puncture and the patient was treated with 3 intramuscular injections of 2.4 million units of penicillin $G$, at weekly intervals. As headaches persisted, a bone scintigraphy was performed and showed multiple skull hyperactive lesions. Skin lesions and headaches disappeared 6 weeks later without any additional treatment.

\section{DISCUSSION:}

Bone involvement is usually seen in tertiary and congenital syphilis but is rare in secondary syphilis. Most of the cases affect HIV-positive patients. Periostitis is the most common bone lesion of secondary syphilis, located most often in the tibiae, followed by the skull. Osteolytic lesions are infrequent and essentially involve the skull and the sternoclavicular joints. Skull lesions affect most frequently frontal and parietal bones. Bone scintigraphy seems to be the most sensitive examination to detect syphilitic lesions. No consensus exists for treatment of
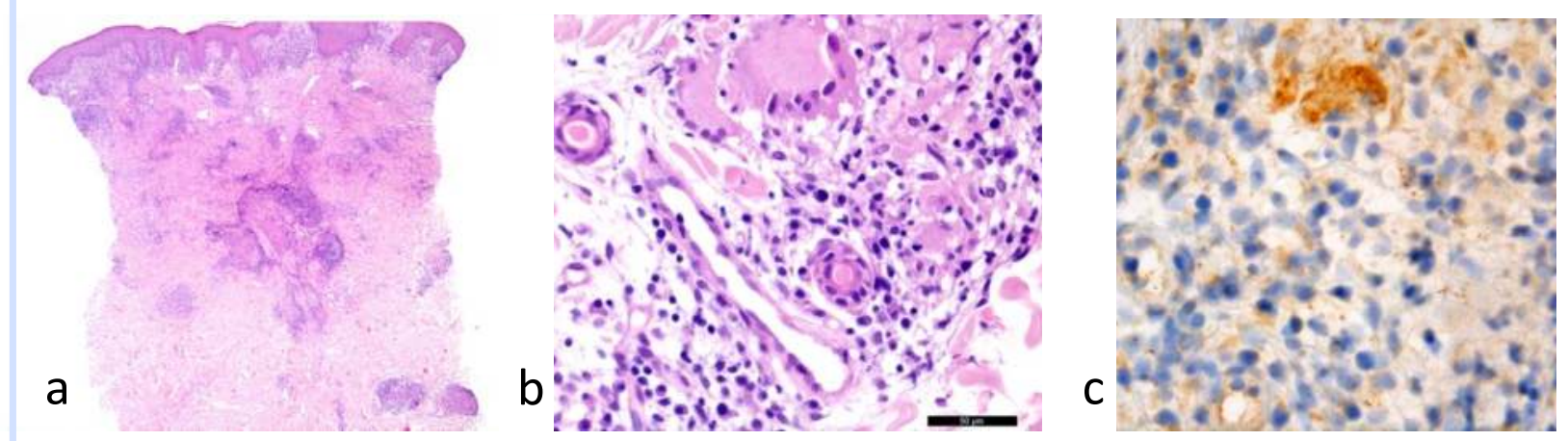

Biopsy of a cutaneous lesion (a) with zoom on a granuloma (b). Treponema immunohistochemistry (c).

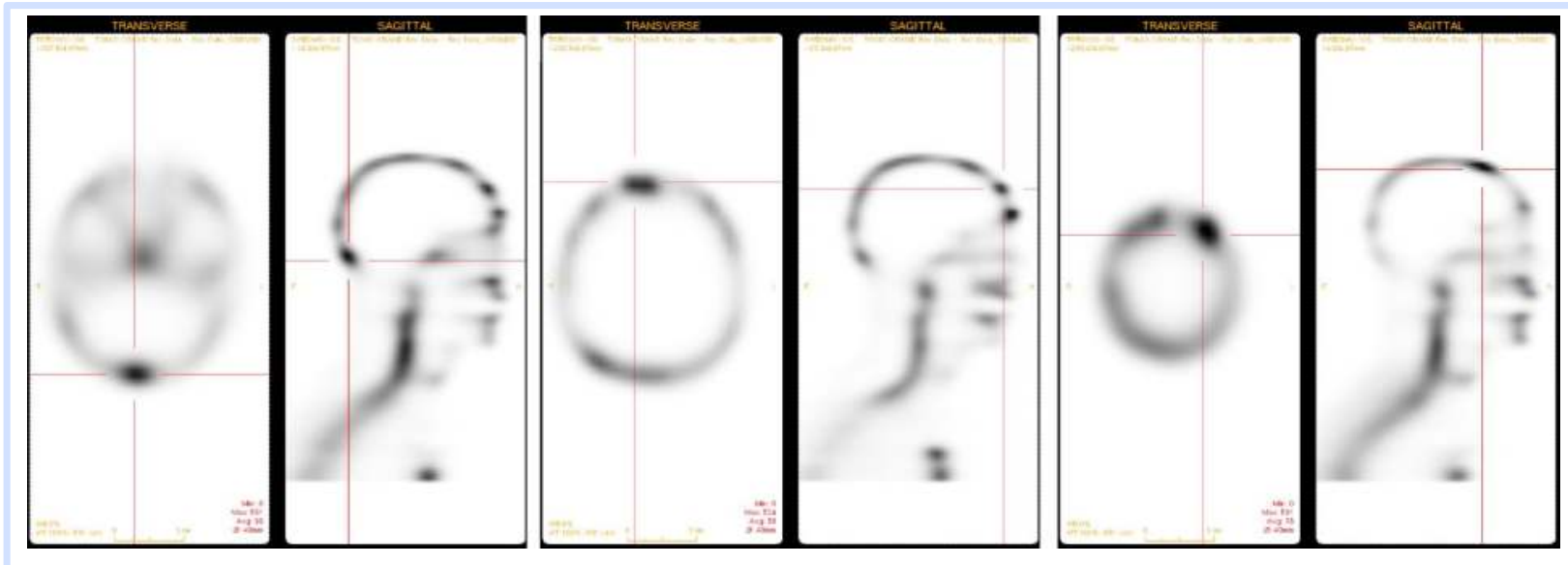

Bone scan highlighting multiple hyperactive skull lesions. bone involvement in secondary syphilis. Clinical presentation with large erythemato-squamous plaques is unusual. Additionally, treponema immunohistochemistry was positive only in the granulomas within deep dermis, whereas epidermis and superficial dermis were surprisingly unaffected.

\section{CONCLUSION}

In a context of secondary syphilis, headaches can be due to bone lesions of the skull. In cutaneous biopsy, absence of treponema beneath the epidermis by immunohistochemistry does not rule out syphilis. Careful examination of the deep dermis is necessary, especially in the presence of deep-seated granulomas.


Cutaneous lesions before (on the left) and six weeks after the treatment (on the right). 